\title{
New indications on the Galactic bulge IMF by microlensing surveys
}

\author{
L. Mancini ${ }^{\dagger}$ and S. Calchi Novati \\ Dipartimento di Fisica "E.R. Caianiello", Università di Salerno, Baronissi, Italy \\ Istituto Nazionale di Fisica Nucleare, sez. Napoli, Italy
}

\begin{abstract}
Basing on recent microlensing observations, we analyse the mass spectrum of the Galactic bulge stellar population and study the slope of the initial mass function.
\end{abstract}

Keywords. gravitational lensing, Galaxy: bulge, stars: mass function

\section{Introduction}

Today the microlensing observations have become a useful tool in searching for nonluminous compact objects. Originally conceived to establish whether the halo of the Milky Way is composed of this type of objects, these experiments revealed sensibility also to the faint objects of other Galactic components (bulge, disk, spiral arms), as well as the halo and the components of other target galaxies (essentially Magellanic Clouds and M31). Actually, the l.o.s. towards the Galactic bulge have shown to be as a real horn of plenty of microlensing events (the number of events revealed is more than two orders of magnitude greater than those of the other targets). However, the microlensing parameters are better constrained if we use selected subsamples of the events formed just by clump giant sources. This choice is justified to avoid the blending effects and turns to be crucial to study the physical features of the Bulge, like structure and composition.

\section{Optical depth and normalization}

The present analysis is based on the data reported by the EROS (Hamadache et al. 2006), OGLE (Sumi et al. 2006) and MACHO (Popowski et al. 2005) collaborations. A remarkable fact is the very good agreement among the results of three experimental groups on the estimate of the optical depth, in accord also with theoretical expectations. Taking into account this fact, we choose to normalize the bulge density to the observed value of the optical depth. As a fiducial value we take the result reported by the MACHO collaboration towards the CGR (Central Galactic Region), namely $\tau=2.17 \times 10^{-6}$ for $(l, b)=1.50^{\circ}, 2.68^{\circ}$ (Popowski et al. 2005). For our fiducial model, the model E2 of Stanek et al. (1997), this gives us a central bulge density of $\rho_{0}=9.6 \times 10^{9} \mathrm{M}_{\odot} \mathrm{kpc}^{-3}$, for a bulge mass out to $2.5 \mathrm{kpc}$ of $1.5 \times 10^{10} \mathrm{M}_{\odot}$.

\section{The Galactic bulge IMF}

The mass function of the Bulge stars has been studied by many authors in different mass ranges. Usually, the data are fitted with a power law $\xi(m) \propto m^{-\alpha}$, where $\alpha$ is the slope of the function. Zoccali et al. (2006) found $\alpha=1.3$ for stars having a mass in

$\dagger$ Present address: Dipartimento di Fisica "E.R. Caianiello" - Università degli Studi di Salerno - Via S. Allende - I 84081 Baronissi (SA) - Italy. - email: lmancini@physics.unisa.it 


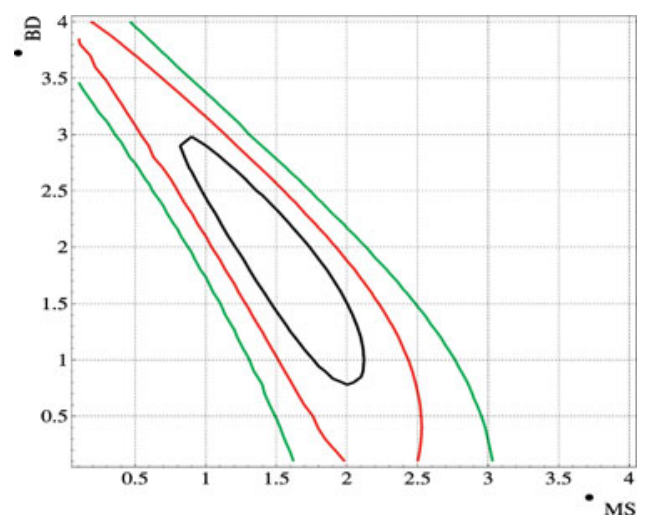

Figure 1. Probability isocontours with $34 \%, 68 \%$ and $90 \%$ region in the $\alpha_{\mathrm{BD}}, \alpha_{\mathrm{MS}}$ plane. $\alpha_{\mathrm{BD}}$, $\alpha_{\mathrm{MS}}$ are the slopes of the power law IMF of the Galactic bulge lenses, in the brown dwarf and main sequence range, respectively. Here the set of 42 events reported by the MACHO collaboration in the CGR is considered.

the range $[0.15-1] \mathrm{M}_{\odot}$. Gould (2000) assumed that all the stars with mass above $1 \mathrm{M}_{\odot}$ could be considered as remnants (white dwarfs, neutron stars, black holes). In order to calculate the mass fraction of each of these components, he proposed $\alpha=2$ for the slope of IMF. Then, Han \& Gould (2003) and Wood \& Mao (2005) extended the analysis of microlening data including brown dwarfs and main sequence stars with very low mass.

Here we introduce two parameters, the slopes $\alpha_{\mathrm{BD}}$ and $\alpha_{\mathrm{MS}}$ in the mass ranges $[0.01-$ $0.08] \mathrm{M}_{\odot}$ and $[0.08-1.0] \mathrm{M}_{\odot}$ respectively. We want to constrain them by using the maximum likelihood method. As a starting point we evaluate the microlensing differential rate with respect to the event duration, along each of the revealed event directions. Then, taking into account the efficiency, we normalize the rate to unity so to get the probability distribution for the event duration. We take as free parameters the exponents of the IMF in the brown dwarfs and main sequence ranges, $\alpha_{\mathrm{BD}}, \alpha_{\mathrm{MS}}$, that we want to estimate. Finally, we evaluate the likelihood, summing up the disc and the bulge contributions, and for each the contribution of the brown dwarfs, main sequence and remnants lens populations. In Fig. 1 we show, in the $\alpha_{\mathrm{BD}}, \alpha_{\mathrm{MS}}$ parameter space, the contours of equal probability for the MACHO data set for the 42 events reported within the CGR (that, roughly, can be considered as a rather homogeneous region), for our fiducial configuration. The data get to reasonably constrain the IMF slope in the main sequence range, whereas the probability turns out to be strongly degenerate along the direction of the brown dwarfs slope. At maximum probability we get the value $\alpha_{\mathrm{MS}}=1.6$, correspondingly the average mass is $0.1 \mathrm{M}_{\odot}$, the expected average timescale is $t_{E}=16$ days, and the mass fraction of the different lens population is $\sim(21 \%, 54 \%, 25 \%)$ for brown dwarfs, main sequence, and remnants, respectively (Calchi Novati et al. 2007).

\section{References}

Calchi Novati, S., De Luca, F., \& Jetzer, Ph., et al. 2007, submitted to Astronomy \& Astrophysics Gould, A. 2000, Astrophysical Journal 535, 928

Hamadache, C., Le Guillou, L., \& Tisserand, P., et al. 2006, Astronomy \& Astrophysics 454, 185 Han, C. \& Gould, A. 2003, Astrophysical Journal 592, 172

Popowski, P., Griest, K., \& Thomas, C. L., et al. 2005, Astrophysical Journal 631, 879

Stanek, K. Z., Udalski, A., \& Szymanski, M., et al. 1997, Astrophysical Journal 477, 163

Sumi, T., Woźniak, P. R., \& Udalski, A., et al. 2006, Astrophysical Journal 636, 240

Wood, A. \& Mao, S. 2005, Monthly Notice of the Royal Astronomical Society 362, 945

Zoccali, M., Cassisi, S., \& Frogel, J. A. et al. 2000, Astrophysical Journal 530, 418 